MEI

II, vol. 2

$\mathrm{n}^{0} 2$

\title{
Exposiciones digitales y reutilización: aplicación del software libre Omeka para la publicación estructurada
}

\author{
Tomás Saorín \\ Universidad de Murcia
}

\section{Resumen}

El artículo contextualiza las exposiciones digitales en el marco de la difusión digital de las colecciones de museos y patrimonio, presentando las funcionalidades del software libre Omeka para la gestión especializadas de este tipo de contenidos, a través de una combinación ágil de gestor de colecciones digitales vinculado a generador de exposiciones. El sistema usa metadatos normalizados y aplica OAI-PMH, de forma que permite la reutilización y distribución de contenidos en la web. También se analizan las ventajas del software como servicio (SaaS) para el caso de Omeka Net.

\section{Palabras clave}

Repositorios; Colecciones digitales; Omeka; Acceso abierto; Reutilización; Exposiciones virtuales.

Digital Exhibition and reuse : implementation of free software for publishing structured Omeka

Abstract

This paper contextualizes digital exhibitions under digital dissemination of museum collections and heritage, presenting the functionalities of open software Omeka for specialized management of this type of content, through an agile combination of a digital collection manager connected to a exhibitions generator. System uses normalized metadata and applies OAI-PMH, so that allows reuse and content distribution in the Web. Also is analyzed the advantages of software as a service (SaaS) for Omeka Net.

\section{Keywords}

Repositories; Digital collections; Omeka; Open access; Reuse; virtual exhibitions.

\section{Introducción}

Un buen número de instituciones de todo tipo, desde los grandes a los pequeños museos, pasando por las entidades locales, fundaciones, centros de arte, universidades, bibliotecas, archivos, centros de investigación o sindicatos, están siendo productores de exposiciones. La exposición es un medio de comunicación de masas singular, cuyo formato está vinculado a un período temporal limitado, un espacio físico adaptado y flexible (especialmente en la itinerancia) y a un conjunto variable de objetos, mensajes y elementos museográficos.

Esta comunicación con objetos, vinculada a la ampliación de los mensajes difundidos desde los propios museos o desde instituciones que adoptan circunstancialmente los mecanismos del museo, ha conocido un desarrollo 
importante, del que nos proponemos estudiar algunos aspectos de su presencia digital. Cuando una institución adopta el contexto expositivo, "ofrece la posibilidad de establecer una lectura diferente, no documental como en una biblioteca, sino objetual, con todas las posibilidades que de ello emanan, pero con el mismo carácter informativo y documental. También es objetual, es decir, única y original, incluso la contextualización documental que el archivo de la institución puede ofrecer sobre el objeto, su historia, ingreso en la institución, los artistas, personajes relacionados, etcétera” (Gutiérrez Usillos, 2010).

La exposición en línea, derivada habitualmente de una exposición física, es un campo en el que se advierten las debilidades de una gestión de información cortoplacista, discontinua y, sobre todo, gestionada como un subproducto, sin una apuesta por generar valor por sí misma. Sin embargo, podemos aplicar sobre ellas el enfoque de la estandarización, la reutilización y la consistencia informativa, para posibilitar otro recurso más de información con capacidad de generar valor en el estado actual de las redes. También abordaremos cómo conseguir un modelo de publicación sostenible y flexible para diferentes entornos organizativos y condiciones tecnológicas.

La exposición suele ser considerada como el instrumento de comunicación más sobresaliente del museo. También es un formato cada vez más usado por instituciones no estrictamente museísticas. Por medio de la exposición se presenta al público el patrimonio que conserva y se transmiten mensajes (valores, conocimientos, enfoques) utilizando objetos a modo de emisarios culturales, vehículos de ideas y sensaciones. Además de la exposición estable, la colección, cada vez tienen más importancia las exposiciones temporales para captar atención pública o construir nuevos discursos.

Los museos son conscientes además de que tienen que encajar su estrategia digital con el resto de sus políticas, e incluso en muchos casos, considerarla un canal preferente y con capacidad de impacto comunicativo ${ }^{1}$. Otras veces la web es tratada como un mero producto de apoyo que tan sólo resuelve una función auxiliar.

\section{Exposiciones en línea: enfoques y sostenibilidad}

Queremos circunscribirnos a las exposiciones en línea, contemplando sólo tangencialmente otros ámbitos con los que están estrechamente vinculadas, como la web del museo o el acceso al catálogo de la colección estable. Habitualmente encontramos los términos "exposición virtual", "exposición digital" o "exposición online". También es frecuente encontrarnos simplemente ante un subsite para la exposición, formando parte de la web institucional, o un microsite propio, realizados con tecnologías e identidad visual específicas. ¿Cómo afecta el medio digital al universo de las exposiciones? Podemos identificar los siguientes aspectos:

- La presencia web es uno de los elementos necesarios al producir y distribuir una exposición temporal. 
- Dentro del espacio físico de las exposiciones también están presentes contenidos digitales creativos, basados en la interacción in situ en dispositivos adaptados al uso público (pantallas táctiles, interfaces simplificadas, navegación cerrada, etc.).

- Existen oportunidades de crear espacios de información en la red con significados autónomos, sin vinculación a una exposición convencional en las salas del museo.

- El concepto de colección digital tiende a encajarse en moldes normalizados (modelos conceptuales, reglas de catalogación, metadatos y vocabularios estandarizados), susceptibles de actuar en red, y por lo tanto abiertos a posibilidades de reutilización (Hillman; Coyle; Phipps; Dunsire 2010).

- Los museos pueden necesitar construir, también en la red, una "experiencia" singular de sus exposiciones.

- Empieza a experimentarse también con la posibilidad de acceso a información inalámbrica in situ, con smartphones del propio visitante, ampliando el espacio físico de la exposición con elementos de realidad aumentada.

Frente a la colección (una base de datos), la exposición es un documento (una narración). Carreras y Munilla (2005, p. 84) consideran que una distinción a la hora de crear los contenidos de una institución de la memoria se encuentra en "la forma de priorizar el objeto frente a la narración o viceversa, tanto en una exposición temporal como en la muestra de la colección permanente.

Esta dicotomía, que ya está presente en la representación presencial de las muestras, también provoca un tratamiento diferenciado en los espacios virtuales”. La exposición es pues un discurso museográfico articulado a partir de, por ejemplo, un tema central, un objeto central singular, ordenamiento cronológico, preguntas y respuestas, una historia en primera persona, jerarquía científica, analogías, etc.

Para las exposiciones en línea podemos diferenciar en primera instancia entre aquellas que cumplen una función complementaria de una exposición real, y aquellas que tienen existencia autónoma (producidas y materializadas para su consumo primario en el entorno digital).

En el primer caso, suele plantearse que contenidos virtuales y reales no coincidan completamente, para que cada uno de ellos favorezca la visita del otro. En el segundo, se afronta el reto de trasladar a una publicación digital el carácter de experiencia valiosa asociado a la visita de exposiciones. Encontramos un territorio problemático en la definición de estrategias acertadas que equilibren las tensiones entre experiencias e información. De un lado entran en juego los conceptos de escasez, atención e inmersión, y del otro los de abundancia, acceso y contextualización.

Los modelos de complementariedad pueden ser (Carreras; Munilla, 2005, p. 99-102): 
- Modelo de difusión, en el que la exposición virtual no compite con la visita, y es por tanto la única alternativa de acceso. En este caso, la función prioritaria del recurso virtual es ampliar la difusión de la muestra presencial, en otras palabras llegar allí donde no puede la exposición real.

- Modelo de conexión, de forma que hay contenidos a los que sólo se accede digitalmente. La información digital también sirve para contextualizar antes y después de la visita. Esto permite que la exposición sea un contínuum, que incorpore tanto la parte virtual, como la presencial, con un discurso expositivo o hilo conductor común.

- Modelo de superposición, donde el espacio virtual incorpora la mayor parte del contenido de la exposición

Otro elemento próximo a la exposición en línea es de visita virtual, el cual es un lugar común en los sitios webs de museos, así como en el imaginario de la comunicación social. Consideramos muy devaluado este concepto de "visita virtual" cuando se asimila a "recorrido virtual", que simula el espacio de las salas y dependencias. Sus potencialidades sólo tienen efecto cuando se trata, efectivamente, de enriquecer información espacial relevante (arquitectura, urbanismo).

En general, pueden ser proyectos que exigen fuertes inversiones en tecnologías, poco adecuados al crecimiento informativo y, por lo tanto, poco sostenibles como estrategia digital. Además pueden competir en la asignación de fondos para tecnologías que ofrecerían mayor rentabilidad, como las presentadas en este trabajo, que aportan una propuesta consistente de valor, costes razonables y son informativamente sostenibles.

Conviene pues plantearse cómo se usa la presencia virtual antes, durante y posteriormente a la visita real. ¿Es compatible que cumplan simultáneamente el papel de folleto, guía, ampliación y memoria? ¿Cuántos recursos se pondrán en cada una de estas facetas, y qué valores esperamos construir desde ellas? Como instituciones de la memoria es posible que el apremio del calendario de eventos, el marketing de visitas y el impacto mediático, nos esté haciendo dejar de lado la dimensión "memoria".

Desde el punto de vista de la rentabilidad a largo plazo, al preguntarnos sobre qué perdura del esfuerzo intelectual y de la inversión de una exposición, nos encontramos con que fundamentalmente permanecerá en forma de documento convencional; el catálogo de la exposición, en formato impreso o, a veces, en formato digital empaquetado (generalmente pdf o flash).

Nuestra percepción, desde el punto de vista de la eficacia informativa y de producción, es que la elaboración digital de exposiciones puede basarse en patrones de piezas sencillas combinadas, con alta coherencia en las fuentes de información usadas, sobre los que podrán desarrollarse diferentes niveles complementarios de experimentación y creatividad, con un criterio más utilitario y de experiencia de usuario rica (juegos, animaciones). 
La presencia digital alrededor de una exposición podría concebirse como capas de valor, construidas progresivamente en función de los tiempos y costes de producción. Desde el punto de vista de la gestión de información los aspectos ineludibles serán los que garanticen una información de la exposición usable, bien posicionada en buscadores, producida con agilidad, con riqueza en información contextual de los objetos y capacidad de ser usada en diferentes contextos informativos. Otros elementos con función estética, didáctica o de espectáculo han de quedar supeditados a la previa realización de las funciones informativas estructurales básicas.

\section{Las exposiciones no son islas}

El que las exposiciones temporales sean proyectos bastante autónomos en su gestión y producción no nos debe hacer perder de vista el museo como sistema de información. En los últimos años se ha avanzado en la definición de unos principios internacionales de documentación en museos (Icom-Cidoc, 2007) y un modelo conceptual, Cidoc-Icom Conceptual Reference Model (Crofts; Doerr; Gill; Stead; Stiff, 2003-2010) que comprenden tanto la catalogación de los objetos culturales, como su gestión integral en el seno de las instituciones de conservación y difusión. En los principios se remarca la vinculación de la información generada en las exposiciones con el sistema de información:

- Deberán incorporarse al sistema de información los contenidos resultado de investigaciones donde estén implicadas las piezas o de la elaboración de catálogos de exposiciones, así como su cita mediante código único de identificación en trabajos publicados.

- El sistema permitirá integrar la información existente sobre las colecciones y objetos a otros recursos como materiales didácticos o exposiciones en línea.

La progresiva adopción de estándares de metadatos está permitiendo un aumento significativo de la disponibilidad de recursos digitales descriptivos y visuales de las colecciones de museos, que podría permitir una eclosión de la información del patrimonio cultural, en combinación con las estrategias digitales del resto de instituciones de la memoria, en el contexto de las organizaciones-red y los formatos abiertos y enlazados (Saorín Pérez, 2011b, p. 18).

La complejidad de la gestión integral del sistema de información del museo, volcado hacia sus procesos internos, puede limitar la capacidad de innovar en la web, en la información pública. La dificultad de mantener coherencia en la estrategia digital entre todos los departamentos y proyectos, puede provocar que las exposiciones (o las actividades didácticas, por poner otro ejemplo próximo) adopten soluciones web coyunturales de muy diferente naturaleza, por lo general sin conexión con las bases de datos del museo. No es nuestro propósito defender la uniformidad de tratamiento para todos los tipos de contenidos, pero sí la exigencia de que todas las herramientas de información se conciban más allá de su utilidad inmediata, teniendo en cuenta la sostenibilidad, reutilización y coherencia. 
Por eso nos parece relevante conectar la exposición virtual con los objetos culturales digitales que conforman la colección. Una exposición conecta, relaciona e interpreta piezas y objetos, a los que añade un discurso. La exposición en línea puede actuar como una vía de acceso singular al conjunto más amplio que supone el sistema de información del museo. El usuario llegaría a la colección desde la exposición.

Y si hasta ahora hemos hablado de islas para la relación entre exposición y museo, la metáfora puede ampliarse para hablar de redes de museos y redes de fuentes de información culturales. Por eso, cuando hablamos de "objeto cultural" nos situamos más allá de las colecciones de los museos, aunque ellas constituyan el núcleo. La producción de exposiciones a menudo exige relacionar información procedente de múltiples sistemas de información poco conectados: patrimonio monumental, artístico, científico, bibliográfico o documental. Aquí se abre el bullicioso campo de la interconexión del patrimonio cultural, y las confluencias entre archivos, bibliotecas y museos, del que Europeana es un buen exponente.

Por lo tanto, en el ecosistema de los objetos culturales, las exposiciones serán a su vez consideradas un objeto cultural, el cual transforma el contexto de los objetos que contiene más allá de su mera descripción, incorporándolo a un acto de comunicación.

Cuando los sitios webs de museos y exposiciones - y también muchos portales sobre patrimonio cultural - son islas o silos informativos que apuntan únicamente a sus propios contenidos, dificultan ser enlazados, remezclados y apropiados por los usuarios de la red. Una web de museo, por abundante y central que sea, estará perdiendo capacidad si no está conectada con otras fuentes digitales de información digital (Hernández Carrascal, 2010, p. 152). Y no se trata únicamente de un problema de tecnología o formatos, sino fundamentalmente de estrategia para transformar la organización y liberar el valor organizacional de la información hasta entonces confinada en silos de datos y contenido.

Podríamos hablar también de la incorporación de una arquitectura de la participación para conectar con prácticas digitales extendidas (etiquetado social, comentarios, etc.). Pero, el elemento esencial será cómo se potencia la navegación entre objetos de sus propias colecciones y los de otras colecciones, o cómo plantea el sistema relaciones interesantes entre objetos. La reutilización de datos culturales pasa, entre otros, por la extensión de las prácticas de catalogación de objetos culturales más allá de los sistemas exclusivamente museísticos y su formalización mediante los recursos de la web semántica (RDF, SKOS), ya que "el movimiento Open data revolucionará en pocos años el mundo del acceso a los datos" (Peset; Ferrer; Ferrer-Sapena; SubiratsColl, 2011, p. 172).

\section{Omeka y la publicación de contenidos}

Omeka es una aplicación de software libre orientada a resolver las necesidades básicas de publicación de contenidos digitales en $\mathrm{OAI} / \mathrm{PMH}$, y construir de forma sencilla exposiciones en línea o publicaciones a partir de estos recursos. Permite la publicación 
de un repositorio de objetos digitales organizados en colecciones, los cuales pueden complementariamente integrarse en diferentes exposiciones en línea. Es al mismo tiempo un repositorio digital y un generador de exposiciones.

Es un desarrollo de Roy Rosenzweig Center for History and New Media (George Mason University), al igual que Zotero, y funciona bajo el entorno Php-MySQL, al igual que OJS, OCS, Drupal, Wordpress, Joomla o Moodle. Podríamos decir que Omeka podría ser "el wordpress para las exposiciones". Una plataforma de productividad rápida, basadas en tecnologías ampliamente difundidas, y con un excelente rendimiento para las necesidades básicas de publicación en web de contenidos culturales convencionales.

Omeka se anuncia como "A serious web publishing platform" para museos y bibiotecas, investigadores y archiveros, educadores y entusiastas. El adjetivo "serious" quiere decir que el peso de los proyectos está en los propios contenidos, y no en la plataforma en el que se sostienen. Su modelo de publicación lo constituyen "complex narratives and share rich collections". Estas narrativas (las exposiciones) se apoyan en la identificación individual de cada uno de los objetos usados (ítems).

En resumen, sus características son²:

- Facilidad de instalación (Entorno php-mysql).

- Flexibilidad y extensibilidad (Zend Framework, APIs documentadas y módulos desarrollados por la comunidad).

- Estructura Dublin Core.

- Interoperatibilidad con otros sistemas de colecciones digitales.

- Adecuado a las normas W3C de accesibilidad.

- Almacena todo tipo de ficheros (imágenes, video, audio, documentos multipágina y PDFs, presentaciones Power Point, etc.)

- Los objetos o ítems individuales pueden contener múltiples ficheros digitales asociados y el sistema puede manejar grandes cantidades de metadatos y ficheros.

- Los datos pueden compartirse en Atom, DCMES-XML, JSON, y RSS2.

- Selección de tema visual.

- Sindicación.

- Etiquetado.

- Creación de colecciones.

- Mapeo de colecciones.

- Creación de campos controlados por listas.

- Sistema de búsqueda en las colecciones incorporado.

Omeka puede ser una forma de resolver con limpieza el ámbito de las exposiciones digitales, usando un molde y una pauta. La aplicación de Omeka permitirá producir 
múltiples difusiones en línea de recursos culturales (o exposiciones en línea) con un coste muy reducido en formación y presupuesto. Nos permite apoyarnos en estándares que dan valor, aumentan la accesibilidad y orientan nuestra acción hacia los contenidos y no el formato. Además permite reutilizar una tecnología en sucesivas exposiciones, por lo que su aprendizaje supone una inversión en productividad a corto y medio plazo.

¿Cómo encaja Omeka en la diversidad de sistemas para la gestión de contenidos web? El siguiente diagrama dibuja con claridad su posición intermedia entre los Sistemas de Gestión de Contenidos de propósito general (CMS como Wordpress o Drupal), los sistemas complejos de Biblioteca Digital o Repositorios (como Dspace o Fedora), y los sistemas de gestión integral de colecciones de museos (como Domus o TMS).

Omeka pretende ser una solución eficaz, barata y sencilla, para un tipo de publicación en línea de calidad, basada en la descripción individualizada de objetos (catalogación de objetos culturales) basada en estándares como Dublin Core y OAI-PMH. Es, por lo tanto, un gestor de contenidos especializado.

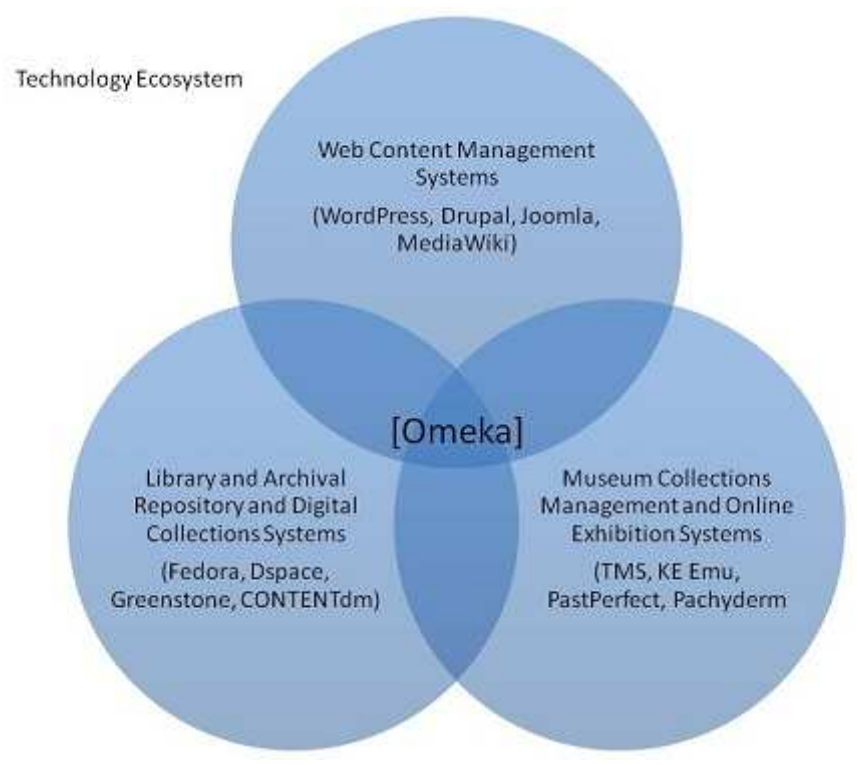

Fig. 1. Ecosistema tecnológico de Omeka (fuente: Blog de Omeka.org (Scheinfeldt, 2010)

Hemos de remarcar que no podrá buscarse en Omeka un software libre para la gestión integral de la colección, sino tan sólo para la capa de comunicación pública de colecciones (Catálogo y Exposiciones). En la esfera del software parece prometedor el enfoque de CollectiveAccess ${ }^{3}$, una aplicación web altamente configurable para catalogar colecciones de museos, archivos y colecciones digitales. Se distribuye bajo liencia GPL, soporta una variedad suficiente de conjuntos de metadatos (Dublin Core, PBCore y SPECTRUM), captura de datos externos y formatos de medios. También ofrece soporte multilingüe. 
Los usuarios potenciales de Omeka pueden ser tanto creadores de contenidos (Grupos de investigación, profesores, investigadores, colectivos sociales), como bibliotecarios y archiveros (cuyos fondos también son susceptibles de explotarse mediante exposiciones) así como profesionales de los museos (Conservadores, Comisarios, Diseñadores, Departamentos de Educación y proveedores de servicios tecnológicos).

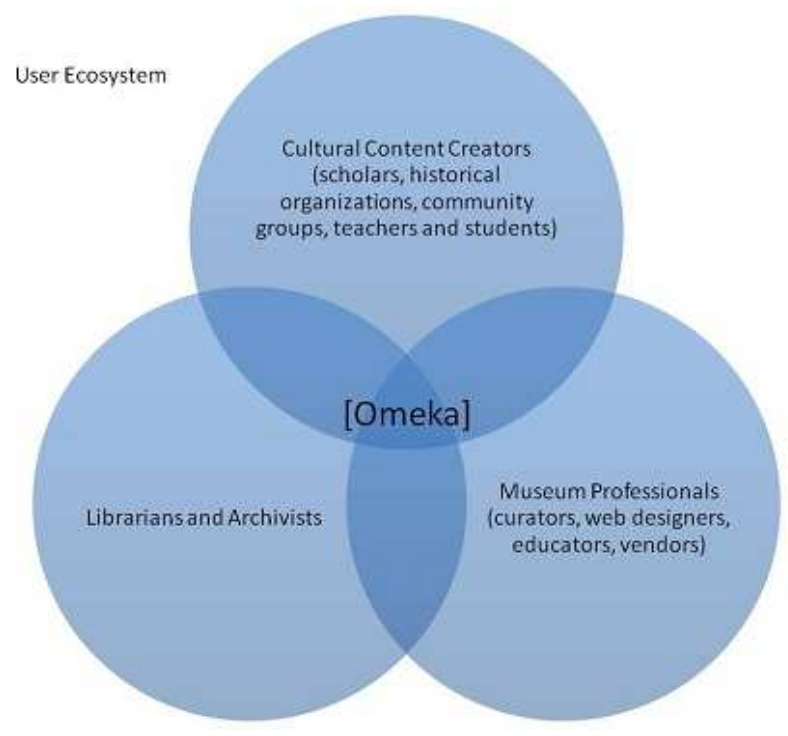

Fig. 2: Ecosistema de usuarios de Omeka fuente: Blog de Omeka.org (Scheinfeldt, 2010)

Desde el punto de vista del modelo de negocio y servicios para instituciones culturales, este tipo de plataformas son una oportunidad para adecuar la actividad de las empresas encargadas de la producción de exposiciones, el diseño gráfico y los elementos de comunicación. Tomando como punto de partida las prestaciones de una plataforma se consigue caer en errores de diseño (accesibilidad, navegación, contexto, posicionamiento, etc.).

Además se consigue reducir todos los costes de producción, asegurar unos mínimos de calidad y compatibilidad, y acumular la experiencia y reutilizar las mejoras añadidas ad-hoc en los siguientes proyectos. Se trata de un marco ventajoso tanto para empresas como para los centros culturales contratantes.

\section{Exposiciones y sitios web realizados con Omeka}

Podemos encontrar ejemplos en los que se aprecia la alta calidad que puede conseguirse trabajando a partir de una plataforma abierta, la cual aporta la estructura básica (Repositorio y Exposiciones) y permite añadir el "look and feel" del diseño, y las mejoras singulares necesarias para cada proyecto.

Algunos ejemplos de interés son: 
- Lincoln at 200, sobre el bicentario de Abraham Lincoln (Chicago History Museum y Newberry Library). http://lincolnat200.org

- Memorial Stadium (University of Minnesota). http://brickhouse.lib.umn.edu

- Making the history of 1989 (Roy Rosenzweig Center for History \& New Media). http://chnm.gmu.edu/1989

- Digital Amherst (Jones Library of Amherst). http://www.digitalamherst.org

En estos ejemplos puede verse una de las características de la plataforma: en función de la estrategia de comunicación adoptada en su realización, el sitio web puede responder principalmente a:

a) El catálogo de una exposición (Lincoln at 200).

b) Una publicación digital (Making de history of 1989).

c) Un repositorio o catálogo público de una colección (Digital Amherst). No se elabora una exposición, sino que tan sólo se ofrece un repositorio.

Aunque la mayor parte de los ejemplos de sitios con contenido y acabado avanzados provienen de los Estados Unidos de América, es de señalar que una de las exposiciones virtuales desarrolladas por Europeana, sobre Art Nouveau ${ }^{5}$, está desarrollada con Omeka, lo cual es un indicio a tomar en consideración.
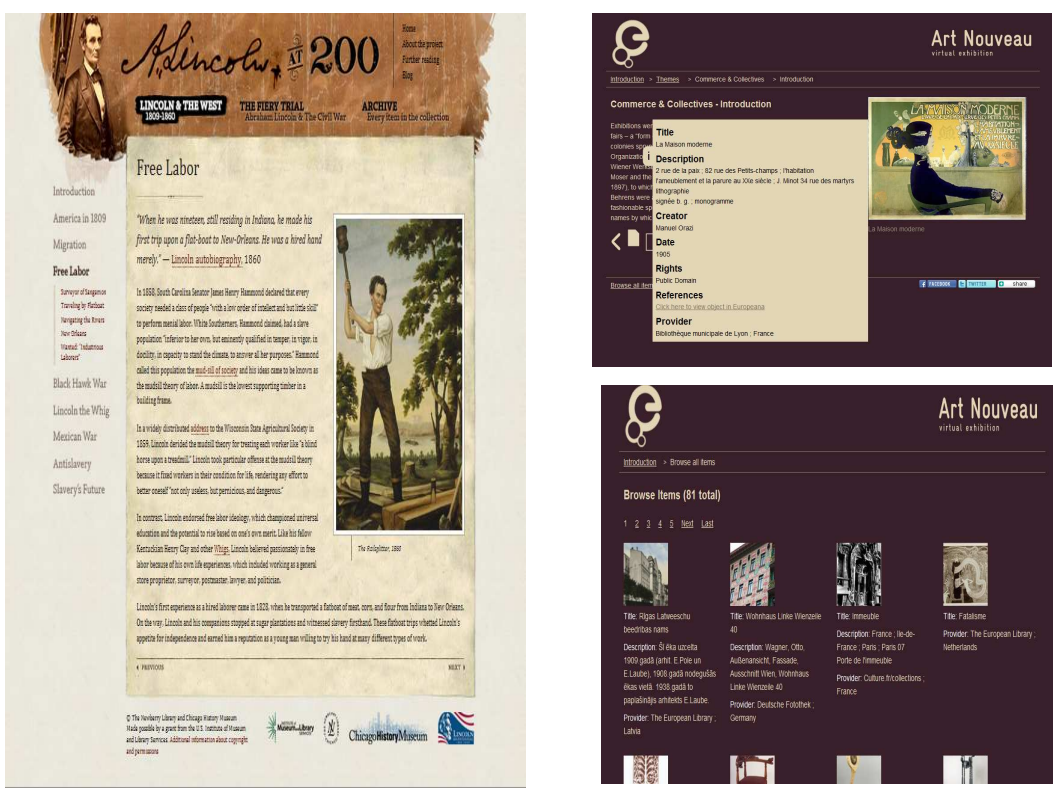

Fig. 3: Imágenes de los sitios web de la exposiciones "Lincoln at 200” y “Art Nouveau” 
También consideramos de interés el análisis de caso del proceso de selección y adaptación de plataforma para la gestión de colecciones digitales del Metropolitan New York Library Council, partiendo de experiencias previas tanto en proyectos con ContentDM como con Wordpress, que se materializa en el proyecto colaborativo digitalMETRO, realizado sobre Omeka (Kucsma; Reiss; Sidman, 2010).

\section{Estructura y funcionamiento de Omeka}

Consideremos a Omeka no como un repositorio, sino un gestor de colecciones digitales, en donde "la organización y creación de los mecanismos de acceso o la gestión de metadatos se benefician de la combinación de los procesos de publicación digital, y de los principios de gestión de información”, donde además se tiende a "configurar espacios de colaboración, mediante el acceso y la gestión de colecciones distribuidas" (Tramullas, 2005)

Para trabajar con Omeka lo primero será comprender las tres entidades que se gestionan:

- Items (Objetos culturales)

- Collections (Agrupaciones ad hoc)

- Exhibits (Exposiciones, que reutilizan los ítems)

Los dos primeros son los que encontramos en muchos repositorios digitales. La institución alimentará un repositorio en donde se almacenan los elementos individuales, los cuales por lo general contienen una representación visual del mismo (Por ejemplo, una o varias fotos de una escultura). La institución agrupa, conforme a su criterio propio, los ítems en colecciones. Por ejemplo, algunos museos agrupan por "salas", "plantas", "Sección arte precolombino" o "Donación McAnudo".

Estos dos elementos crean el nivel de REPOSITORIO. En la base de una exposición en Omeka habrá siempre un repositorio sencillo, que usa elementos de descripción normalizados y que es accesible vía OAI-PMH. Lo interesante es que Omeka añade además una capa de publicación a partir de los objetos del repositorio, permitiendo construir una publicación, que proporciona una experiencia de lectura mucho más rica y contextualizada. Omeka permite hacer el catálogo de la exposición, pero de forma estructurada y reutilizable a largo plazo.

Cuando creamos una EXHIBIT vamos a crear un nuevo recurso de información, con una estructura jerárquica y con un estilo visual propio. Es importante este último punto, puesto que permite diferenciar entre el repositorio y la exposición, incorporando a ésta una mayor calidad gráfica y de diseño, así como cualquier otra mejora al nivel de presentación.

Una exposición se compone, en Omeka, de: 
- Título global (y unos metadatos asociados) con una url propia.

- Un tema visual asociado, que puede ser diferente del usado en el repositorio.

- Una estructura jerárquica:

o Secciones, o grandes bloques en los que se estructura el catálogo o la exposición. Están compuestas de páginas.

o Páginas: Contenido final en el que junto al texto explicativo se incluyen ítems del repositorio (objetos catalogados), aplicando una de las plantillas de maquetación de página propuestas.

o Las secciones necesitan contener páginas para poder visualizarse.

- Cada objeto usado en las páginas permitirá el enlace a su ficha del catálogo, en donde se contiene su descripción individualizada. La exposición no se desvincula del catálogo, se superpone a él aportando valor de comunicación.
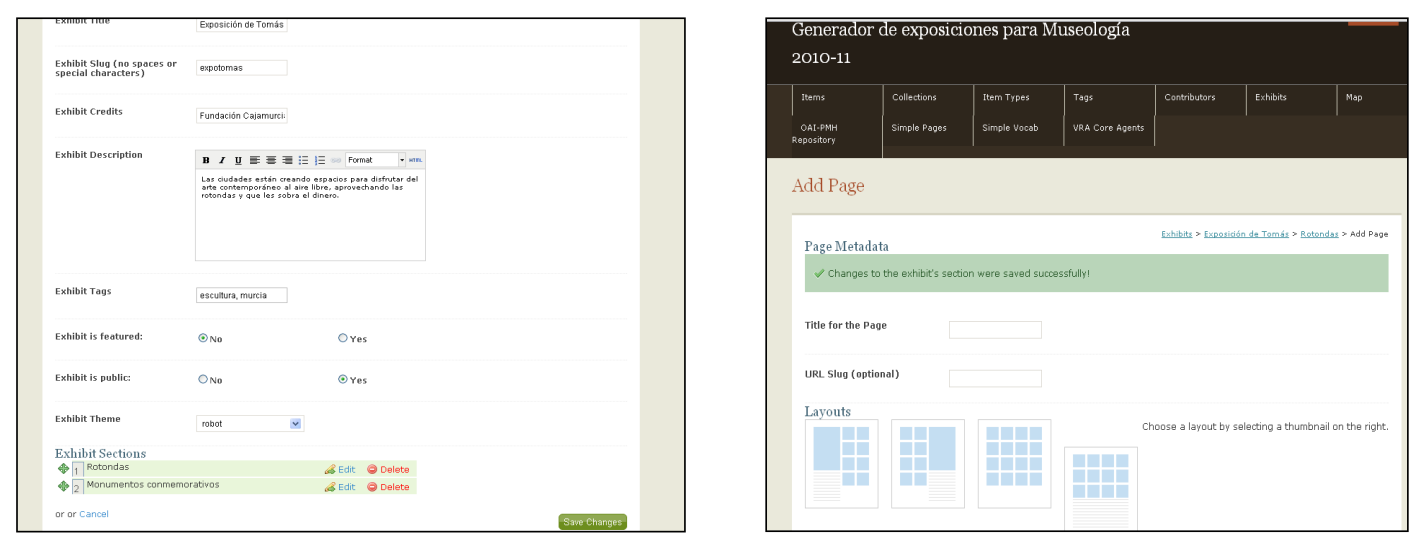

Figura 4: Ejemplos del entorno de gestión de contenidos con Omeka

Un sistema montado con Omeka es de rápida puesta en marcha, pero su explotación en contextos reales siempre va a requerir al menos la personalización del tema visual.

Se trata del mismo modelo que usan casi todas las plataformas de gestión de contenidos, basadas en la separación entre el contenido y la presentación.

Esto permite separar el trabajo de generación del contenido, de carácter interno y especializado de la institución documental, de la subcontratación del diseño web usando estándares y aplicándolo a través de plantillas normalizadas.

Una consideración final es la de que una institución que cuenta con Omeka ya funcionando, al producir una exposición en línea, tendrá que delegar en empresas de diseño seleccionadas para crear un acabado final atractivo, basado en la modificación de los temas y plantillas visuales de Omeka, pero siempre basado en los estándares de hojas de estilo y accesibilidad. 
Omeka proporciona utilidades básicas de gestión de usuarios, perfiles de acceso y administración. Pero, frente a otros sistemas de gestión de contenidos, presenta mayores rigideces para su ampliación. Por ejemplo, el versionado multilingüe, la navegación por tags o la definición espacios de consulta privados. Frente a ellos, limita el número de opciones a gestionar y presenta un paquete coherente de funcionalidades que solucionan necesidades frecuentes y esenciales.

Dado que definir políticas de gestión de información y publicación digital es susceptible de errores de enfoque y materialización, una plataforma base reduce el margen de error. Omeka simplifica las elecciones, aunque a cambio se paga un precio acotando el campo a la creatividad ${ }^{6}$.

\section{Generación de exposiciones reutilizables y abiertas}

Si se reduce la vertiente digital de las exposiciones a, en el mejor de los casos, una versión electrónica del catálogo-libro, estaremos perdiendo una de las externalidades clave de la web 2.0: la reutilización.

Internet es una red abierta, y hay que entender nuestros contenidos desde esa apertura: "No podemos considerar los repositorios como infraestructuras encerradas en sí mismas. No es comparable el valor que se pueda añadir a una colección de documentos generados por una organización en concreto con las posibilidades de explotación que pueden tener estos contenidos desde la óptica de la agregación y con la capacidad de generar servicios a partir de la suma de miles de documentos provenientes de distintos repositorios. Es por ello que no podemos hablar de servicios sobre los repositorios sin contemplar su capacidad de interoperar, entendiendo la interoperabilidad como la posibilidad de intercambiar datos entre distintos sistemas." (Prats; Rovira, 2009, p. 102)

La creación de un catálogo exige aplicar una plantilla de descripción (metadatos). Omeka está diseñado para aplicar en todo momento Dublín Core, al que se pueden añadir otros elementos descriptivos decididos por cada institución. Permite incorporar campos propios, adaptables a las prácticas descriptivas de un museo.

En particular existen dos plugins que permiten extender los metadatos:

- Dublín Core Extended, que amplia los 15 campos clásicos.

- VRA Core, que amplia los campos al modelo de la Visual Resources Association, para la descripción de imágenes.

Omeka trata de mantener un equilibrio entre simplicidad y capacidad de extensión. Otros plugins nos permitirán, por ejemplo:

- Integración con Zotero, añadiendo metadatos COins.

- Geolocalización y construcción de líneas de tiempo. 
- Construir vocabularios simples.

- Definir tipos de licencia Creative Commons.

- Salida para sindicación en Atom o MediaRSS.

- Funciones sociales como Comentarios (Contribution) para aportación de contenidos por usuarios, o My Omeka, para personalizar accesos, página de contacto, o funciones para el Social bookmarking.

- OAI-PMH Harvester.

- Anotación de imágenes y establecimiento de relaciones entre items.

- Utilidades, como importación CSV, importación EAD, Códigos de barras y etiquetas para objetos.

- Integración con aplicaciones web como Google Docs o Dropbox.

Los registros de un sistema con Omeka pueden ser recolectados en OAI/PMH, añadiendo un elemento más de calidad y reconocimiento.

Usar Omeka de forma complementaria a los sistemas corporativos utilizados en la actualidad (por ejemplo Domus) puede ayudar a la visibilidad en Hispana o Europeana. Si rediseñar y adaptar infraestructuras complejas y en producción es un obstáculo, e implica tiempo y recursos, Omeka puede funcionar como pasarela de transición, ya que no puede olvidarse que para "resolver el problema de la utilización de distintos formatos de metadatos, incluso de carácter local, en especial en los sectores de museos y archivos, mediante la implementación de los principales esquemas y la utilización de procesos automáticos de mapeo y pasarelas entre ellos" será un aspecto crítico la utilización de una arquitectura y estándares de metadatos interoperables de Europeana. (Alonso Arévalo; Subirats Coll; Martínez Conde, 2008, p. 47)

A menudo vemos la reutilización únicamente como un asunto de terceras partes. Sin embargo sucede a menudo que las instituciones y sus sistemas funcionan como agentes externos. Los datos abiertos lo son también entre departamentos. Por eso es valiosa la política de Omeka de "re-purposing": "Enter or import item metadata once, and use items and metadata in multiple instances across website. Items may appear in multiple exhibits".

También podemos señalar la existencia de una oferta de plataformas de gestión de colecciones digitales como servicio, que permite a las instituciones culturales contratar gestionar servicios en línea administrados. Esta corriente de software como servicio, junto al coste afinado del cloud computing, acercan soluciones tecnológicas viables a pequeñas y medianas instituciones, eliminando el riesgo de obsolescencia y los costes de gestionar los propios proyectos tecnológicos. Usando estándares, y garantizando la apertura y seguridad de los datos y objetos digitales almacenados, se suavizan las tensiones producidas por la gestión remota de recursos de información institucionales. Cualquier institución puede, a un precio escalable y conocido, disponer de herramientas de catalogación, carga, transformación, control de acceso, publicación y compartir en línea. 
Omeka puede utilizarse a través de:

Omeka.net, siguiendo el modelo de otras plataformas de software libre. Puede usarse totalmente en línea, mediante cuentas con diferentes prestaciones (sites, plugins, espacio, etc.), incluyendo una totalmente gratuita.

\section{Conclusiones}

Las exposiciones tienen un enorme potencial de comunicación, dado su alto nivel de elaboración, a través de su selección de objetos, temática singular y formas de expresión narrativas. Por otro lado los repositorios y bases de datos no están orientados al consumo directo por un sector amplio de usuarios. Cada uno cumple su papel y, a menudo, coexisten paralelos, sin elementos de conexión entre ellos.

Omeka permite orientar la generación de exposiciones en línea de forma que se combinen ambos enfoques, de forma que la creación de una publicación estructurada esté vinculada a la descripción individual de cada pieza. Además ofrece un patrón para que la producción técnica de exposiciones en línea sea económica y los esfuerzos puedan, por lo tanto, centrarse en la calidad de los contenidos.

Tampoco es un valor menor la disponibilidad inmediata de colecciones accesibles mediante OAI-PMH, separando la administración de la plataforma de otras tareas de administración de museos y centros culturales. Una plataforma eficiente libera recursos y capacidades para generar una propuesta de valor específica en cada institución.

En el extremo de la simplificción están las plataformas SaaS, que simplifican la toma de decisiones, y conectan de forma muy directa con la obtención de valor tangible. Son una opción a considerar seriamente para superar las barreras de entrada.

Sería deseable, no obstante, una mayor participación en las comunidades de software libre, y no actuar meramente como usuarios, desarrollando módulos útiles o ajustando otros a los contextos regionales o nacionales.

Aún vemos limitado el alcance del movimiento de software libre en instituciones culturales (como es caso de SLIC en España ${ }^{7}$ ), dado que su éxito supone la capacidad de gestión y aplicación de estrategias a medio plazo, que pueden fácilmente exceder sus conocimientos y estructuras de gestión. Deseamos que maduren comunidades activas en el desarrollo con software libre, con proyectos en los que se visualicen buenas prácticas y actúen como atractores en este campo. Aunque la escala del sector limita la posibilidad de productos comerciales, para el éxito del "open source" no basta con aplicar un ramillete de tecnologías accesibles y sin coste, hay que incorporar la colaboración en las prácticas informativas y tejer mejores redes (Wilde; Mann, 2010). 
Dado que la mayoría de las instituciones europeas del patrimonio son de pequeñas dimensiones, el informe europeo Digicult resaltaba la necesidad de crear organizaciones de apoyo e infraestructuras digitales para aprovechar las oportunidades de la revolución digital. La producción de exposiciones en línea podría ser más eficiente si se aplicasen sobre ellas modelos estructurados de publicación digital: los costes de producción se concentrarían en la elaboración del mensaje específico de la exposición y en la aplicación de un estilo visual, los cuales descansarían sobre una plataforma de gestión de contenidos especializada, con posibilidad de normalizar los metadatos y de permitir apertura para recolectores, buscadores y mashups.

Omeka, y otras iniciativas basadas en software libre, cumple los objetivos de publicar con calidad, sencillez y proyección hacia el futuro, al separar los datos de los objetos o piezas de la estructura narrativa de la exposición digital. Al mismo tiempo permite trabajar en red, creando plataformas descentralizadas para la generación de exposiciones en redes de museos y centros expositivos.

\section{Bibliografía}

ARÉVALO, J.A.; SUBIRATS COLL, I: CONDE, M.L. (2008). Informe APEI sobre acceso abierto. Gijón: Asociación Profesional de Especialistas en Información.

CARreras Montort, CÉSAR; MUNILla CABrillana, GLÒRIA (2005). Patrimonio digital. UOC.

CROFTS, NICK; DOERR, MARTIN; GILL, TONY; STEAD, STEPHEN; STIFF, MATTHEW. (2003-2005). Definition of the CIDOC Conceptual Reference Model (Version 5.0.2). ICOM/CIDOC CRM Special Interest Group.

Digicult report: technological landscapes for tomorrow's cultural economy; unlocking the value of cultural heritage. (2002). Luxembourg: Office for Official Publications of the European Communities.

DigitalMETRO.

<http://nycdigital.or>

[Consulta: 28 abril 2011].

Europeana, Think Culture.

$<$ http://www.europeana.org>

[Consulta: 28 abril 2011].

GUTIÉRREZ USILLOS, A. (2010) Museología y documentación: Criterios para la definición de un proyecto de documentación en museos. Gijón: Trea.

HERNÁNDEZ CARRASCAL, FRANCISCA (2010). Intercambio de información sobre el patrimonio cultural: La ontología del patrimonio cultural de Cantabria. EN: XII Jornadas de Gestión de la Información: valor económico de la información : mercados, servicios y rentabilidad. SEDIC, pp. 137-154 
HILLMANN, DIANE; COYLE, KAREN; PHIPPS, JON; DUNSIRE, GORDON (2010). RDA Vocabularies: Process, Outcome, Use. En: D-Lib Magazine, Vol. 16, N. 1/2.

<http://www.dlib.org/dlib/january10/hillmann/o1hillmann.htm>

[Consulta: 28 abril 2011].

ICOM-CIDOC (2007). Statement of principles of museum documentation (Version 6.0) Icom-Cidoc.

KUCSMA, JASON; REISS, KEVIN; SIDMAN, ANGELA (2010). "Using Omeka to Build Digital Collections: The METRO Case Study”. En: D-Lib Magazine, Vol. 16, n. 3-4. Disponible en:

<http://www.dlib.org/dlib/march10/kucsma/o3kucsma.htm>

[Consulta: 28 abril 2011].

MATEO RUSILLO, S. (2008). La comunicación global del patrimonio cultural. Gijón: Trea.

Omeka.

<http://www.omeka.org>

[Consulta: 28 abril 2011].

Omeka NET.

<http://www.omeka.net>

[Consulta: 28 abril 2011].

PESET, FERNANDA; FERRER-SAPENA, ANTONIA; SUBIRATS-COLL, IMMA (2011). "Open data y Linked open data: su impacto en el área de bibliotecas y documentación”. En: El profesional de la información, Vol. 20, n. 2, pp. 165-173.

PORTÚS, M. DOLORS; RIUS, TONI; SOLANILLA, LAURA (2009). "La virtualización de las instituciones del patrimonio: navegando por el museo". En: Vives, Josep (coord.) Digitalización del patrimonio: archivos, bibliotecas y museos en la red. UOC.

PRATS, JORDI; ROVIRA, ANNA (2009). "Diseño de un depósito institucional para la difusión de la producción corporativa”. En: Vives, J. (coord.) Digitalización del patrimonio: archivos, bibliotecas y museos en la red. UOC.

SAORÍN PÉREZ, TOMÁS (2011). "La catalogación de objetos culturales y la difusión en red del patrimonio". En: Anuario ThinkEPI, Vol. 5. Disponible en:

<http://www.thinkepi.net/catalogacion-objetos-culturales-difusion-red-patrimoni>

[Consulta: 28 abril 2011].

SAORÍN PÉREZ, TOMÁS (2011). "La difusión y creación de contenidos digitales locales en el ámbito cultural'. En: Saorín Pérez, Tomás; De Haro de San Mateo, $\mathrm{M}^{\mathrm{a}}$ Verónica. Contenidos digitales locales: modelos institucionales y participativos. Madrid: Federación Anabad, pp. 11-26.

SCHEINFELDT, TOM (21 septiembre 2010). “Omeka and its Peers". En: Blog de Omeka.org,. Disponible en:

<http://omeka.org/blog/2010/o9/21/omeka-and-peers>

[Consulta: 28 abril 2011]. 
MEI, II, Vol. 2, nº 2, pág. 46

TRAMULLAS, JESÚS (2005). "Herramientas de software libre para la gestión de contenidos" En: Hipertext.net, n. 3. Disponible en:

<http://hdl.handle.net/10760/8971>

[Consulta: 28 abril 2011].

VRA Core Metadata Set v 4.0,.

<http://www.vraweb.org/projects/vracore4>

[Consulta: 28 abril 2011].

WILDE, E.; MANN, L. (31 marzo 2010) "Open Source Collaboration: New Models for Technology Development in the Museum Community”. En: J. Trant and D. Bearman (eds). Museums and the Web 2010: Proceedings. Toronto: Archives \& Museum Informatics. Disponible en:

<http://www.archimuse.com/mw2010/papers/wilde/wilde.html>

[Consulta: 28 abril 2011].

\section{Notas:}

1. Un excelente ejemplo de ésto sería la estrategia de la Tate Gallery (Tate online strategy 20102012 / John Snack).

<http://www.tate.org.uk/research/tateresearch/tatepapers/10spring/stack.sht>

[Consulta: 28 abril 2011].

2. Omeka feature list.

http://omeka.org/files/docs/Featurelist.pd

3. <http://www.collectiveaccess.org >

[Consulta: 28 abril 2011].

4. Puede realizarse un seguimiento en:

$<$ http://omeka.org/showcase>

[Consulta: 28 abril 2011].

5. <http://exhibitions.europeana.eu/exhibits/show/art-nouveau/introduction>

[Consulta: 28 abril 2011].

6. Lo cual considero positivo cuando la creatividad se convierte en discrecionalidad y ausencia de enfoque, primando valores diferentes a los de una estrategia de información digital coherente y consciente. Es el caso de muchas decisiones de diseño y tecnologías por proveedores de soluciones museográficas o de diseño gráfico.

7. Los Encuentros de Software Libre e Instituciones Culturales (SLIC) van ya por su quinta edición. En una línea parecida encontramos los Encuentros de Centros de Documentación de Arte Contemporáneo. 